\title{
High Accurate Space Telescope Mirror Made by Light and Thermally Stable CFRP*
}

\author{
Jun KOYANAGI**, Yoshihiko ARAO***, Shin UTSUNOMIYA****, \\ Shin-ichi TAKEDA**** and Hiroyuki KAWADA*** \\ ${ }^{* *}$ Institute of Space and Astronautical Science, Japan Aerospace Exploration Agency, \\ Yoshinodai 3-1-1, Sagamihara, Kanagawa 229-8510 Japan \\ E-mail: koyanagi.jun@jaxajp \\ ***Department of Applied Mechanics and Aerospace Engineering, Waseda University, \\ Okubo 3-4-1, Shinjuku, Tokyo 169-8555 Japan \\ **** Aerospace Research and Development Directorate, Japan Aerospace Exploration Agency, \\ Osawa 6-13-1, Mitaka, Tokyo 181-0015 Japan
}

\begin{abstract}
This paper reports a development of space telescope mirror made by light and thermally stable CFRP. We first compare thermal stabilities of a mirror made by CFRP with a conventional that made by glass material. The superior point regarding the thermal stability of the CFRP mirror to the glass mirror is described. One of the most critical issues for CFRP mirror is the mirror-surface roughness deterioration induced by "fiber-print through". We made a prototype mirror consisting of only CFRP with a gel-coating on the mirror surface, addressing the issue. The gel-coated mirror-surface roughness was $20 \mathrm{nmRMS}$ just after fabrication. Durability of the surface roughness under various hostile conditions is examined in the present study.
\end{abstract}

Keywords: CFRP, Telescope Mirror, Surface Roughness, Fiber-print Through, Gel Coating, Durability

\section{Introduction}

One of the main missions of satellite is an observation; there is basically a telescope system inside observing satellites. Mirrors of the telescope system are one of the heaviest parts in the satellites. The maximum weight of the satellite is determined by the launch capacity of the rocket. Of course, the weight of the main mirror is limited but large mirror can facilitate high resolution observation. Hence the materials having both characteristics of high stiffness and light weight are suitable for mirror materials. Carbon fiber reinforced polymeric composite material (CFRP) is superior to not only the specific stiffness also thermal stability as shown in the following chapter so that CFRP might be an alternative material for the satellite mirrors. However, several problems should be solved before applying CFRP to the mirror; one of them is long-term reliability of mirror-surface roughness.

It is reported that there exist a "fiber-print through" problem for CFRP mirror surface ${ }^{(1-3)}$. It is impossible to polish the surface of CFRP like other mirror materials such as glass, $\mathrm{SiC}$, $\mathrm{Be}$ and so on, therefore it is difficult to produce a surface having required accuracy. Typically, the fiber-print through, which is the surface irregularity dominated by fiber alignment near the surface, appears on the CFRP surface even if the CFRP is fabricated by a replica method that is one of most reasonable procedures to make accurate flat surface for

*Received 12 May, 2010 (No. 10-0201) [DOI: 10.1299/jmmp.4.1540]

Copyright $\odot 2010$ by JSME 
general polymeric materials. The fiber-print through critically deteriorates the surface roughness. To address this problem, a lot of works regarding surface treatment have been done in the mean time; one of the most efficient treatment is "gel-coating" ${ }^{(1-3)}$. However, the long-term assurance of the gel-coated surface roughness has not been examined yet nevertheless the CFRP has time-dependent property. There is a possibility that the surface roughness is deteriorated by the time-dependent property. The time-dependent deformation induced by physical ageing, dewater and internal stress relaxation of the matrix may affect the surface roughness; they should be understood.

In the present study, we first demonstrate the thermal stability of CFRP by a thermal radiation analysis. We secondly introduce the fabricating procedure of CFRP prototype mirrors we made. For the CFRP mirrors, the gel-coating is implemented. The CFRP consists of cyanate resin matrix and high modulus carbon fiber. The experimental results regarding long-term durability of the surface accuracy under hostile conditions are then presented.

\section{Thermal stability of CFRP mirrors}

Figure 1 shows an example of model of a telescope system inside satellite. They consist of a main, second and third concave mirrors and first, second and third flat mirrors and a detector. The first flat mirror is at just backside the main mirror. The paths of the observing wave from the third concave mirror to the detector are emphasized in the figure. Note that we do not show the walls of this satellite but the walls surround it in fact. To observe a target, satellites generally round the earth and a heat income direction varies with the relative directions against the sun and the earth. Hence, the temperature distributions in the telescope system vary with time as the satellite structural walls temperatures vary via mainly radiations from the sun and the earth. However, to make the observation accurate, the temperature distributions, particularly a temperature difference existing in individual mirror, is desired to be negligible.

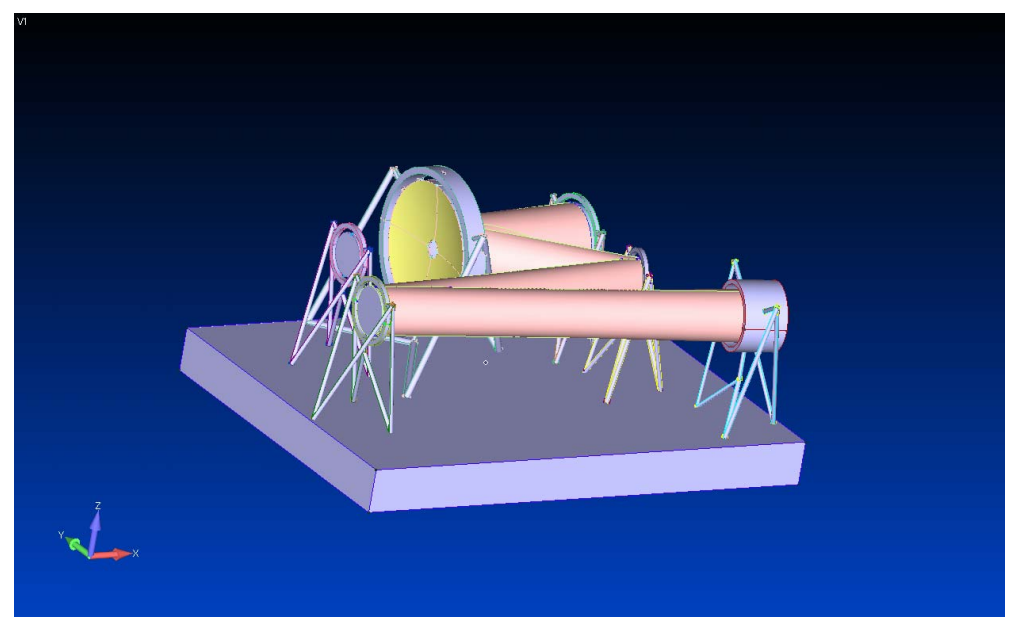

Fig. 1 An example model of telescope system inside satellite

We established a simplified 2D model of the telescope system for thermal analysis as shown in Fig. 2. The model is too simple to simulate quantitative temperature accurately but is useful for a relative comparison of difference of the temperature distributions in CFRP mirrors and conventional glass mirrors. For this model, following conditions are applied. A period of variation of boundary condition is $6000 \mathrm{sec}$, which is assumed that the height of satellite earth-round orbit is approximately $700 \mathrm{~km}$. Based on radiations from the earth and the sun on the orbit, the boundary conditions of the surrounding four walls of this structure can be assumed as shown in Fig. 3. Note that this relationship between wall temperatures 
and time is intended for vernal equinox day. We do not mention here the calculation method to obtain the temperatures shown in Fig. 3. Initial temperature for all nodes $187 \mathrm{~K}$ is given. The four walls are referred as $-\mathrm{x},+\mathrm{x},-\mathrm{y}$ and $+\mathrm{y}$; those in Fig. 2 correspond to those in Fig. 3. Material constants employed in this analysis are shown in Table 1. For the CFRP mirrors, in plane material property is applied along with y-direction and out-of-plane property is applied along with $\mathrm{x}$-direction. The radiation from the cooled detector, which is often required under specified temperature for accurate inferred wave detection, is neglected in the present analysis. There is no air inside the walls; the heat transfers between the mirrors and walls are dominated by thermal radiation phenomenon at vacuumed condition. The heat transfers inside each mirror are simulated. This analysis is performed by ABAQUS ver. 6.7.1.

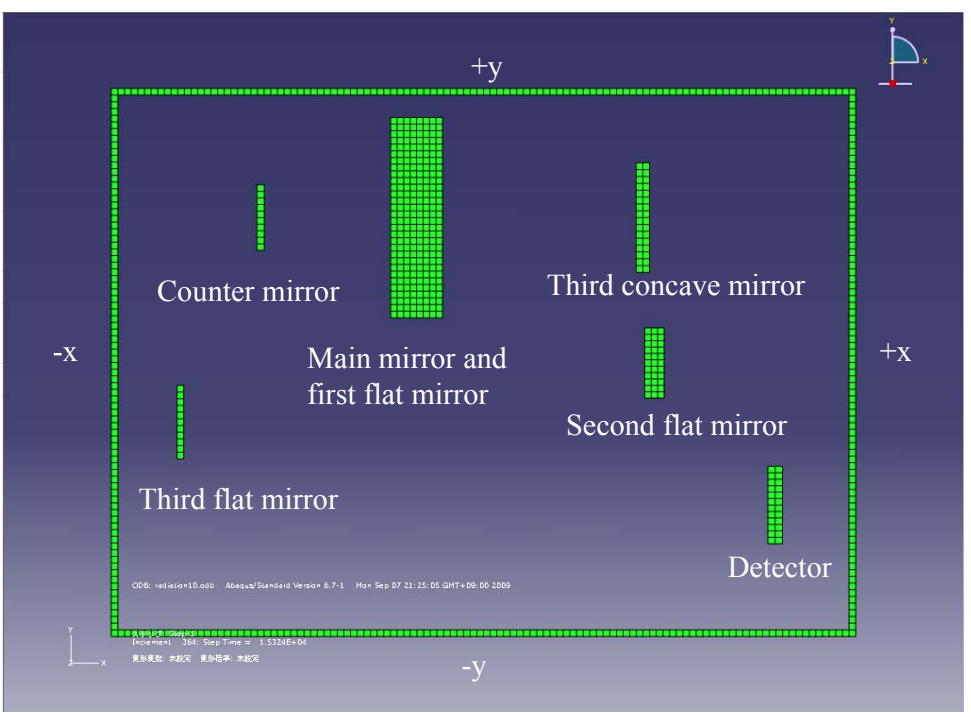

Fig. 2 2D model for thermal analysis inside telescope system

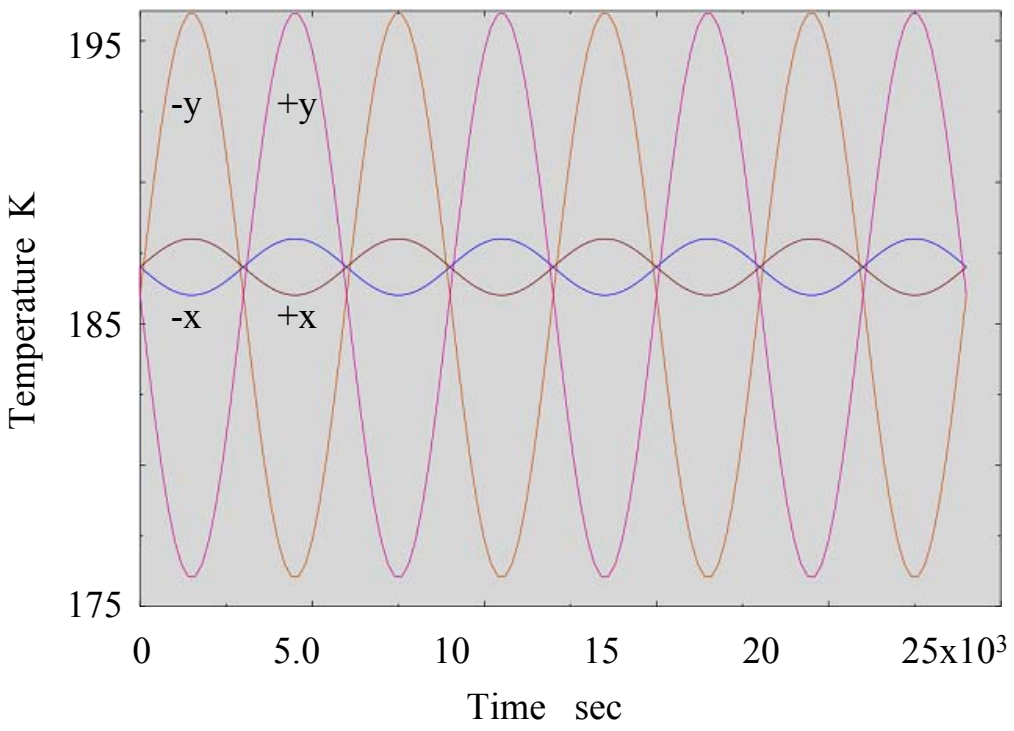

Fig. 3 Boundary conditions for walls of satellite 
Table 1 Material property for thermal analysis

\begin{tabular}{|c|c|c|c|c|}
\hline \multicolumn{2}{|c|}{ Material constants } & CFRP & Glass & unit \\
\hline \multicolumn{2}{|c|}{ Emissivity } & 0.9 & 0.9 & - \\
\hline \multicolumn{2}{|c|}{ Specific heat } & 977 & 755 & $\mathrm{~J} / \mathrm{kgK}$ \\
\hline \multicolumn{2}{|c|}{ Density } & 1.7 & 2.2 & $\mathrm{~g} / \mathrm{cm}^{3}$ \\
\hline Thermal conductivity & $\begin{array}{l}\mathrm{x} \text {-direction } \\
\mathrm{y} \text {-direction }\end{array}$ & $\begin{array}{c}0.20 \\
50\end{array}$ & 1.0 & $\mathrm{~W} / \mathrm{mK}$ \\
\hline \multicolumn{2}{|c|}{ Stephen-Boltzmann constants } & \multicolumn{2}{|c|}{ 5.67E-08 } & $\mathrm{W} / \mathrm{m}^{2} \mathrm{~K}^{4}$ \\
\hline \multicolumn{2}{|c|}{ Absolute zero temperature } & \multicolumn{2}{|c|}{0} & $\mathrm{~K}$ \\
\hline
\end{tabular}

Figure 4 shows a part of analytical results. Those in Fig. 4 are temperature transitions of all nodes for the third flat mirror assuming CFRP and glass materials. Because the required accuracy of third flat mirror is the strictest among all the mirrors from the view point of optics, we usually must check the thermal deformation of the third flat mirror at first. Hence, in the present study, we introduce the temperature of the third flat mirror. As shown in Fig. 4(a), the temperature changes of CFRP can be separated into two groups; all the results are on either of two curves looking thick curves. One consists of the nodes on reflecting face and another consists of the nodes on opposite face. The separation is affected by the low thermal conductivity of the CFRP mirror in x-direction and the high one in y-direction. Comparing with the temperatures of glass material, the temperature scattering is considerably small. It should be also mentioned that the simultaneous temperature change of whole mirror is not fatal in terms of the optics, but it is fatal that each point has individual temperature differently. From this prospective, since the node temperatures for glass material (Fig. 4(b)) scatter widely and change with bad synchronization, the glass mirror is inferior to the CFRP mirror with respect to thermal stability for the present case. Thus, the CFRP mirror is potentially better than conventional glass mirror in terms of not only weight but also thermal stability, depending satellite conditions.

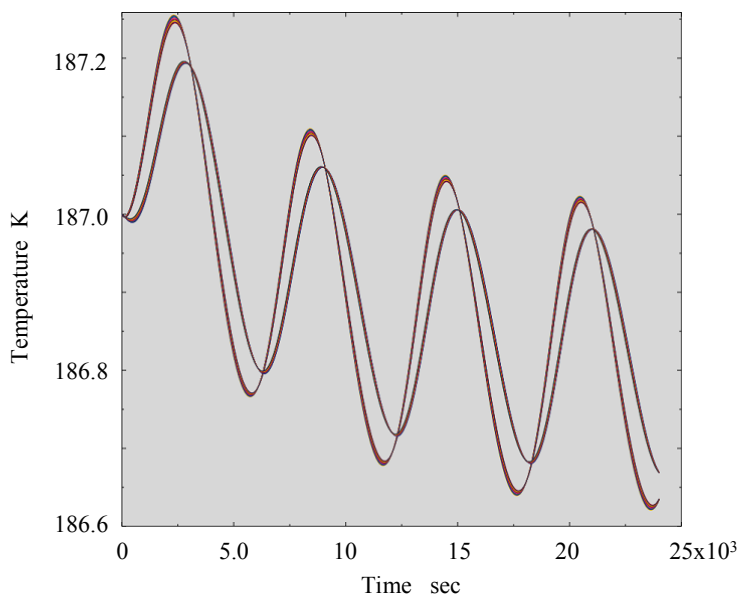

(a) CFRP mirror

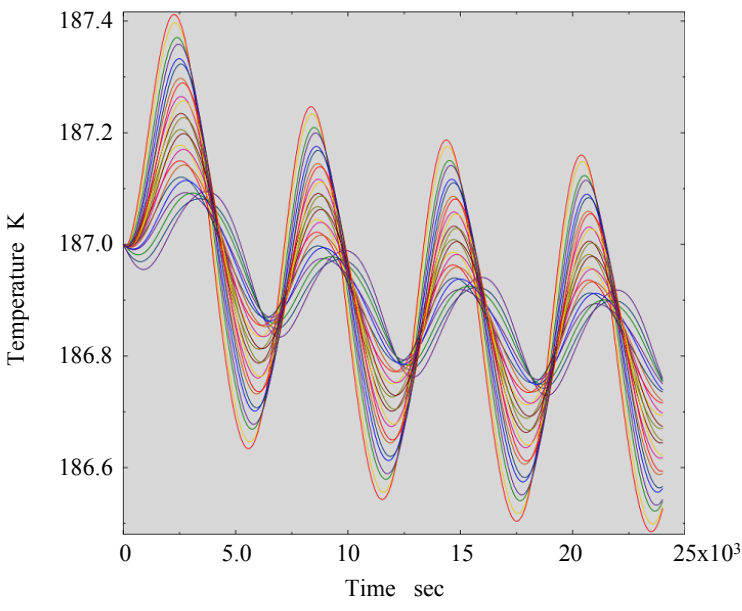

(b) Glass mirror

Fig. 4 Analytical results for (a) CFRP mirror and (b) glass mirror 


\section{Fabrication of CFRP mirrors}

\subsection{Gel-coating for "fiber-print through"}

In advance of introducing the mirror fabrication method, we briefly introduce here "fiber-print through" and the addressing solution we employed in the present study for the fiber-print through problem. Figure 5 shows typical photograph of fiber-print through, taken by SEM. This specimen consists of epoxy resin and carbon fiber. It is fabricated by replica method using optical flat tool in order to make the surface flat. However, on the surface, the pattern of fiber itself appears. From a view of cross-section, a fiber part seems to be lifted up or matrix part seems to be fallen. This is called fiber-print through and it can deteriorate the surface roughness of the CFRP. Although assuming the surface before curing is flat, there are a lot of factors inducing the fiber-print through: a fact that matrix shrinks more than fiber when cooling because of a mismatch of thermal expansion between matrix resin and fiber in radial direction, and curing and physical ageing shrinkages for matrix itself. In the mean time, it has not been reported what is the main factor causing fiber-print through ${ }^{(4)}$ and no article has discussed the inducing mechanism of fiber-print through quantitatively.

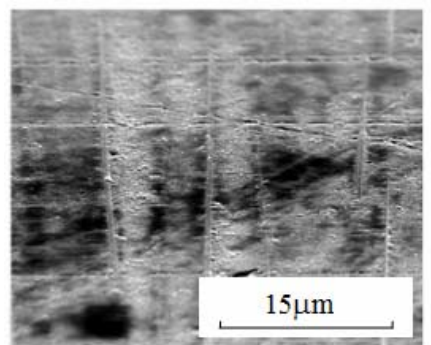

Surface

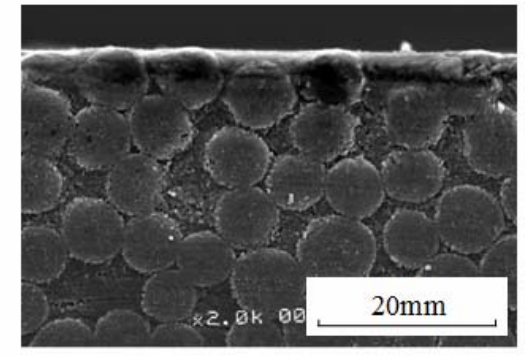

Cross-section

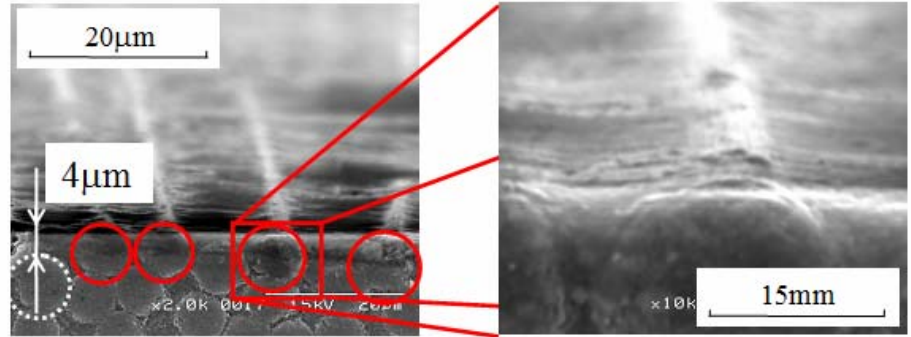

Fig. 5 SEM photograph of CFRP surface (fiber-print through)

As a solution for the fiber-print through problem, there has been "gel-coating" method $^{(1)-(3)}$. The gel-coating is not a critical method exterminating the fiber print through and might, in fact, cause another problem such as a deformation induced by bi-metal effect. However, an appropriate gel-coating can diminish the fiber-print through effectively ${ }^{(1)-(3)}$ even if we do not fully understand the causing mechanism of the fiber-print through. In the present study, we employed the gel-coating and the coating layer is as thin as we can produce stably in order to eliminate another problem occasion. The thickness of gel-coating was about $26 \mu \mathrm{m}$ thickness as shown in Fig. 6. As shown in Fig. 6, it seems that the fiber-print through disappears on the CFRP surface. Note that the gel-coating is implemented onto the surface fabricated by replica method using an optical flat tool and the gel-coating itself is also implemented using the optical flat tool again. By taking this coating treatment, we fabricated the CFRP mirror. 

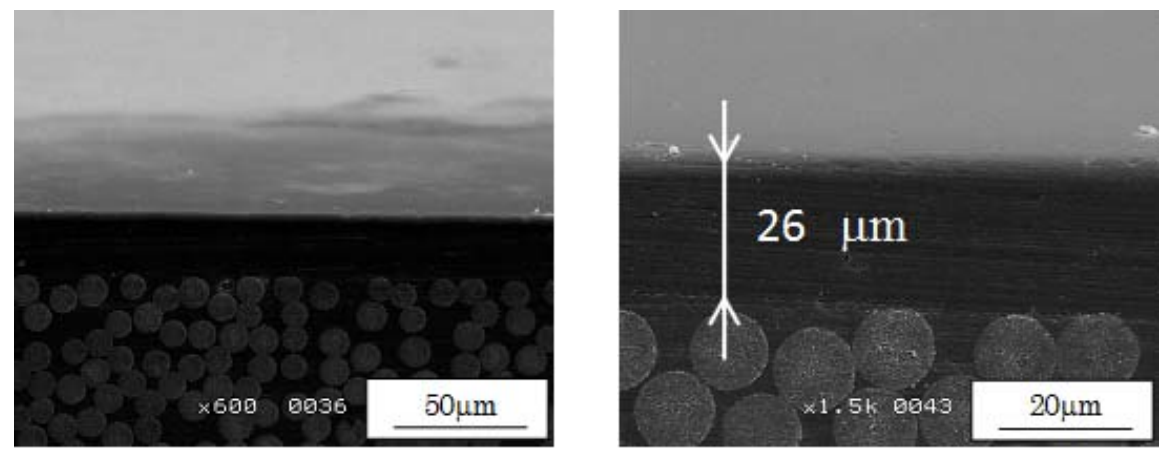

Fig. 6 SEM photograph of gel-coated CFRP surface

\subsection{Fabrication}

Figure 7 shows the flowchart schematically-showing the fabrication procedure for CFRP mirror. First (\#1), we fabricated the front sheet part of the sandwich mirror. Dimensions are $100 \times 100 \times 3$ (thickness). The prepreg (Nippon Graphite Fiber inc.) consists of cyanate resin and pitch-based high modulus carbon fiber. The laminate is fabricated by the curing method the manufacture recommends; $180^{\circ} \mathrm{C} 2$ hours is followed by $232^{\circ} \mathrm{C} 2$ hours. One surface is replicated by an optical flat glass, which only has several nanometers RMS of surface roughness and which is made by ultra low thermal expansion glass material, used as a tool plate of autoclave fabricating method. The stacking sequence of this composite laminate is $\left\{\left[(45 / 0 /-45 / 90)_{\mathrm{S}}\right]_{\mathrm{R} 90}\right\}_{\mathrm{F}}$, which is laminated as follows. We prepare first $200 \times 200$ laminates in $(45 / 0 /-45 / 90)_{\mathrm{s}}$ stacking sequence. We cut this into a pair of $100 \times 200$ dimensions and over-laminate them. But one is rotated $180^{\circ}$ then. Next, we cut again this 16 ply laminate into also a pair of $100 \times 100$ and then they are over-laminated each other again in which one of them is upside down. We refer this stacking sequence as $\left\{\left[(45 / 0 /-45 / 90)_{\mathrm{S}}\right]_{\mathrm{R} 90}\right\}_{\mathrm{F}}$. Since the laminating process is done by human, there exists a possibility that the fiber direction deviates in the laminate. The fiber misalignment can cause unexpected out-of-plane deformation with temperature change which accompanies necessarily with cooling from curing temperature to room temperature. For the purpose of minimizing the fiber misalignment in the laminate, this complicated laminating method is employed in the present study because this laminating method can offset reasonably a local fiber misalignment even when there is a fiber-misaligned ply ${ }^{(5,6)}$.

Second (\#2), we prepared the back sheet part. Dimensions, material and stacking sequence are same as above mentioned front sheet part. Only difference is to use super invar as a tool because of relative easiness for the material acquaintance. Since the optical flat is kept attaching until whole sandwich structure is cured, we need to have another tool for fabricating back sheet laminate. It does not have to be the expensive optical flat glass so we used the super invar tool here. Next (\#3), we prepared a honeycomb core and bonded it between the two laminate sheets. The honeycomb core (Ultracore inc.) consists of also pith-based high modulus carbon fiber and cyanate resin. Dimensions of the core are $100 \mathrm{x}$ $100 \times 20$. In order to bond it and laminate sheets, a cyanate film adhesive is used. The adhesion cure temperature is $120^{\circ} \mathrm{C}$. After these (\#4), the optical flat and super invar tools are removed. And then (\#5), we implemented gel-coating. By using the optical flat again, a very thin vinylester resin layer is composed on the CFRP surface which is produced also by using the optical flat glass again. Here (\#6), a couple of mirrors are implemented buff-polishing in order to try to make more accurate surface. However, since there is a possibility that the buff-polishing works worse, we did not buff-polish another couple of mirrors for the comparison. Consequently, the two of the sandwich mirrors are 
buff-polished and any polishing is not implemented for other two mirrors; we made total four mirrors. Finally (\#7), aluminum evaporation layer is attached on the gel-coated surfaces in order for acquaintance of the sufficient reflectivity for surface-roughness measurement. Following above procedure, we made four sandwich mirrors consisting of only CFRP. The manufactured mirrors are shown in Fig. 8. In the figure, the left two mirrors are buff-polished ones.

1. Fabricating front sheet

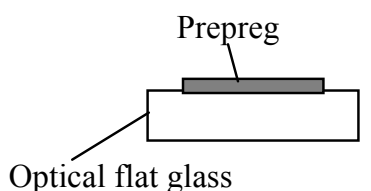

4. Demold

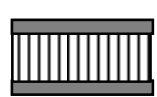

2. Fabricating back sheet

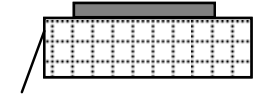

Super invar

5. Gel-coating

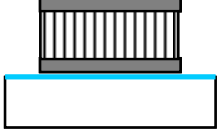

3. Bonding both side simultaneously

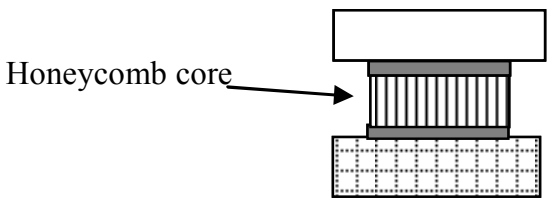

(6. Buff polishing)

7. Aluminum evaporation
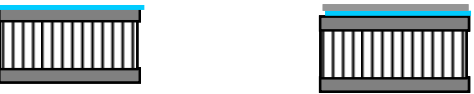

Fig. 7 Fabrication flowchart for CFRP sandwich mirror

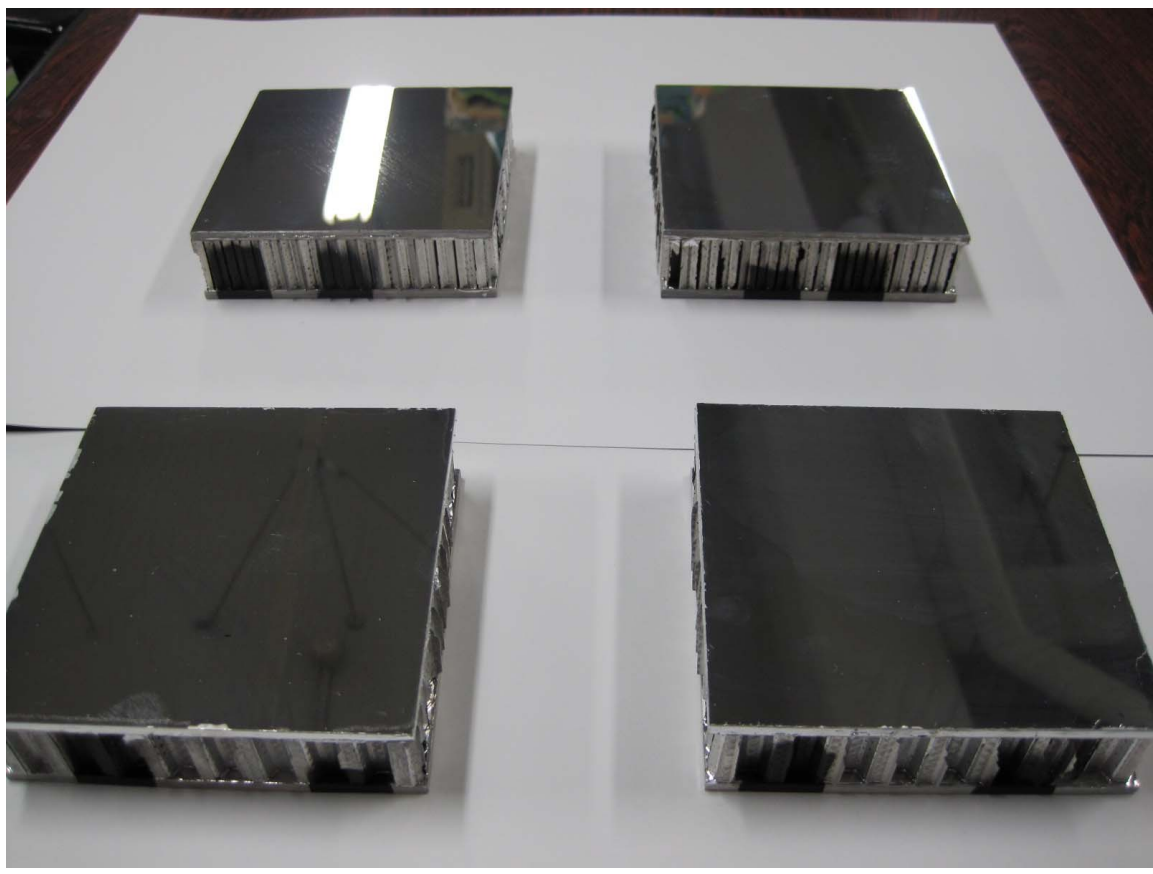

Fig. 8 Photograph of sandwich mirrors consisting of only CFRP

\section{Durability of surface roughness of CFRP mirrors}

CFRP inherently shows a time-dependent property because the matrix resin is time dependent. Once the satellite is launched, there is almost no chance to fix it up. Hence, the long-term assurances for many abilities are required. The long-term durability of the mirror-surface roughness is definitely one of them. In the present study, we examined the 
long-term durability of the mirror surface. We measured surface roughness of above mirrors (approximately $25 \mathrm{~mm}$ square) by Zygo that is a laser interferometer measurement system after the exposure durations under various hostile conditions such as high temperature, high relative humidity and vacuumed conditions. The history of specimen exposure environments and the measurement results of surface roughness after respective exposure are shown in Table 2.

Table 2 Surface roughness measurement results at accumulated various condition durations

\begin{tabular}{|c|c|c|c|c|}
\hline \multirow{2}{*}{ State \# } & \multirow{2}{*}{ Condition } & \multirow{2}{*}{ Duration } & \multicolumn{2}{|c|}{ Surface roughness nmRMS } \\
\hline & & & Buff-polished & Gel-coating only \\
\hline 0 & Initial & - & 20 & 80 \\
\hline 1 & Normal room & 13 days & 50 & 100 \\
\hline 2 & Normal room & 6 days & 40 & 120 \\
\hline 3 & $80^{\circ} \mathrm{C} 95 \% \mathrm{RH}$ & 1 day & 60 & 180 \\
\hline 4 & $20^{\circ} \mathrm{C}$ vacuumed & 1 day & 60 & 180 \\
\hline 5 & Normal room & 8 days & 60 & 180 \\
\hline 6 & $80^{\circ} \mathrm{C}$ vacuumed & 7 days & 60 & 180 \\
\hline
\end{tabular}

As shown in this table, the mirrors were placed at the various conditions and durations from \#1 to \#6 consecutively. Note that the humid conditions are intended for before launch and vacuumed conditions are intended both for dewater condition and space condition. High temperature condition is intended for accelerated testing. As shown in Table 2, the surface roughness was 20 or $80 \mathrm{nmRMS}$ in the initial state. Especially, the surface roughness 20 nmRMS of the buff-polished mirror is expected to be one of the remarkable values in the world for the CFRP mirror. The surface roughness is apparently acceptable to be used for infrared wave mirrors. From $\# 0$ to $\# 2$, it is found that the mirror-surface roughness typically deteriorate with time even at the normal ambient temperature and humidity condition. This is presumably caused by a viscoelastic deformation due to a relaxation of thermal residual stress, a deformation due to a moisture absorption, and physical ageing shrinkage for the matrix respectively. It is hard to understand how critically the respective deformation factor affects because the quantitative study for these deformations of the cyanate resin has not been done enough. For \#3, high temperature and humidity condition is considered to be the most hostile condition, the surface roughness deteriorated relatively rapidly. The cyanate is expected to have a good resistance for moisture absorption; but this result implies the cyanate absorbs water at high temperature and humidity condition even if it is less than that of epoxy resin. After that, the surface roughnesses of all mirrors show constant values no matter what the conditions are. It can be concluded these mirrors are expected to be able to keep their surface roughness after some experiences. Also, since it spent a few days at room temperature from just after fabrication to the first measurement, the surface roughness just after fabrication could be better than 20 nmRMS. That means we could have more accurate mirror if we knew an appropriate preservation way which prevents the mirror surfaces suffering from any aggravations.

We show representative height distributions on the mirror surfaces obtained by Zygo and mention our interpretations here. Figure 9 shows the height distributions of \#0, \#2, \#3 and \#4 for the buff-polished mirror surface. As shown in Fig. 9 (\#0), we can find a surface irregularity with relatively wide pitch wave. This pitch seems to be similar to fiber bundle width. This irregularity is presumably different kind of fiber-print through. In the present study, we refer this pattern as "fiber-bundle print through". We surmise that there are several slight slots between fiber-bundle to fiber bundle in Fig. $9(\# 0)$ and this slot is a resin rich part. These slots seem to become deeper as time progresses even under the normal room 
temperature as shown in (\#2) compared with (\#0). If we assume this might be induced by a physical ageing shrinkage of the cyanate resin, it can be consistent because the assumed resin rich slots can be deeper as resin shrinks. After moisture absorption, the slots seem to be shallow (\#3). Note that the small black mottle in Fig. 9 (\#3) is due to the oxidation of the evaporated aluminum layer while at high temperature and humidity condition and this does not affect the surface-roughness measurements. If we assume again that the slot consists of resin rich part, since polymer materials show usually swelling by moisture absorption, the fact that the moisture makes the slot shallow can be consistent. Further, after dewater by vacuumed condition, these slots get deeper again. It can be assumed the dewater shrinkage of the resin makes the slots deep as well as (\#2). Thus, we found the "fiber-bundle print through" and there are probably resin rich slots. It can be suggested that we have to solve this new fiber-bundle print-through problem in order to obtain better surface further. This is an important aspect to be address in near future.

\section{Conclusion}

In the present study, we reported a progress of development of space telescope mirror made by light and thermally stable CFRP. We exhibited first the thermal stability of assumed CFRP mirror in a satellite by a thermal radiation analysis. We mentioned next "fiber-print through" as a problem of using CFRP for mirrors. The fabrication method considering the problem was described and the surface roughness of the mirrors made by only CFRP was measured. In the initial state, the surface roughness of the sandwich mirror was $20 \mathrm{nmRMS}$, which is expected to be acceptable for the infrared wave mirrors. The long-term durability of the surface roughness is then examined. It was found that the surface roughness was deteriorated at the beginning of the tests and then was kept the constant roughness. We suggest that the "fiber-bundle print through" is one of the factors to be solve in future in order to obtain more accurate CFRP surface.

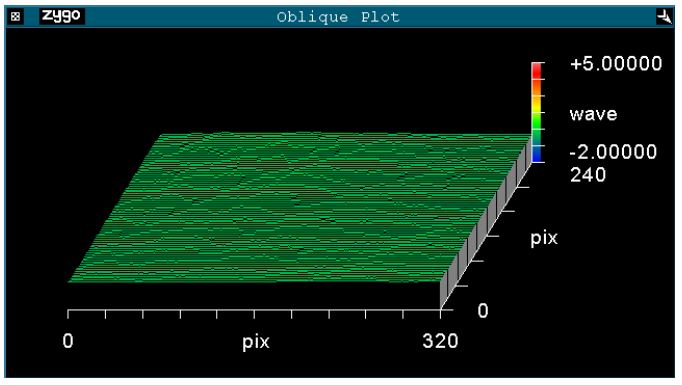

(\#0) Initial condition

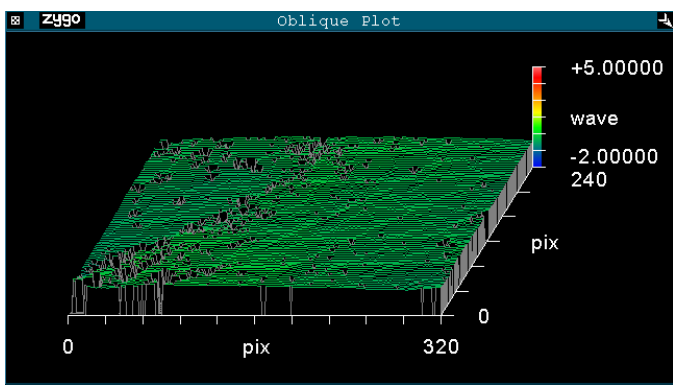

(\#3) 1 day under $80^{\circ} \mathrm{C}, 95 \% \mathrm{RH}$

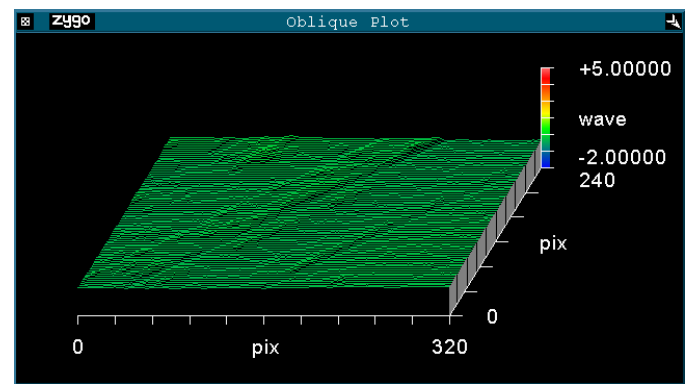

(\#2) Total 19 days under room temperature

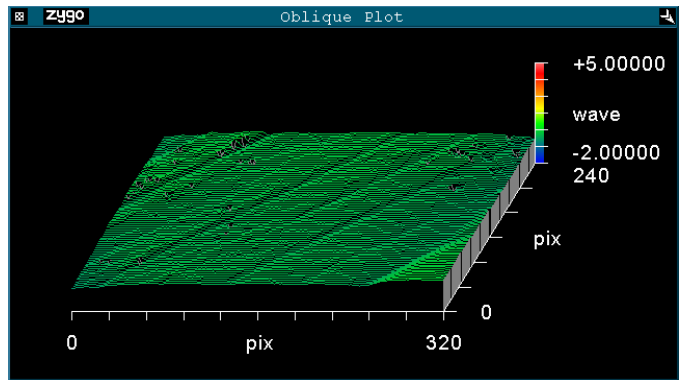

(\#4) 1 day under $20^{\circ} \mathrm{C}$, vacuumed condition

Fig. 9 Height-displacement plot measured by Zygo in representative 1x1 inch area 


\section{Acknowledgments}

This work was supported in part by The Kurata Memorial Hitachi Science and Technology Foundation and by a Grant-in-Aid for Young Scientists (B) from the Ministry of Education, Science, Sports and Culture of Japan.

\section{References}

(1) Massarello, J.J., Hochhalter, J.D., Fuierer, P.A. and. Maji, A.K., Composite mirror replication: curing, coating and polishing, Proceedings of SPIE, Vol. 5868 (2005), pp. 58680.

(2) Hochhalter, J.D., Massarello, J.I., Maji, A.K. and Fuierer, P.A., The origins of fiber print-through in lightweight composite optics, in Novel Optical Systems Design and Optimization IX, Proceedings of SPIE, Vol. 6289 (2006), pp. 628902.

(3) Massarello, J.J., Welsh, J.S., Hochhalter, J.D., Maji, A.K. and Fuierer, P.A., Fiber print-through mitigation technique for composite mirror replication, Optical Engineerig, Vol. 45 (2006), pp. 123401.

(4) Koyanagi, J., Arao, Y., Terada, H., Takeda, Utsunomiya, S. and Kawada, H., Development of Space Telescope Mirror Made by Light and Thermally Stable CFRP, Proceedings of SPIE, Vol. 7522 (2010), pp. 75223.

(5) Arao, Y., Koyanagi, J., Utsunomiya, S. and Kawada, H., Analysis of thermal deformation on honeycomb sandwich CFRP mirror, Mechanics of Advanced Materials and Structures, Vol. 17 (2010), pp. 328-334.

(6) Arao, Y., Koyanagi, J., Terada, H. and Kawada, H.,Time-dependent out-of-plane deformation of symmetric laminate including small fiber misalignment, Conference proceedings of 6th Asian-Australasian Conference on Composite Materials 2008, in CD-ROM, Japan (2008) 